\title{
Use of mycorrhizal fungi for the phytostabilisation of radio-contaminated environment (European project MYRRH): Overview on the scientific achievements
}

\author{
H. Dupré de Boulois ${ }^{1}$, C. Leyval ${ }^{2}$, E.J . J oner ${ }^{2}$, I. J akobsen ${ }^{3}$, B. Chen ${ }^{3}$, \\ P. Roos ${ }^{3}$, I. Thiry ${ }^{4}$, G. Rufyikiri' ${ }^{4}$, B. Delvaux ${ }^{5}$ and S. Declerck ${ }^{1}$ \\ ${ }^{1}$ Université Catholique de Louvain, Mycothèque de l'Université Catholique de Louvain \\ (MUCL), Unité de Microbiologie, 3 place Croix du Sud, 1348 Louvain-la-Neuve, Belgium \\ ${ }^{2}$ LIMOS, UMR 7137 CNRS-UHP Nancy I, Faculté des Sciences, BP. 239, \\ 54506 Vandoeuvre-les-Nancy, Cedex, France \\ ${ }^{3}$ Risø National Laboratoty, Plant Research Department, 4000 Roskilde, Denmark \\ ${ }^{4}$ CEN-SCK, Radiation Protection Research Department, 200 Boeretang, $2400 \mathrm{Mol}$, Belgium \\ ${ }^{5}$ Université Catholique de Louvain, Unité des Sciences du Sol, 2/10 place Croix du Sud, \\ 1348 Louvain-la-Neuve, Belgium
}

\begin{abstract}
Because plants significantly affect radionuclides (RN) cycling and further dispersion into the biosphere, it is important to understand the biological factors influencing RN plant uptake, accumulation and redistribution. In this respect, mycorrhizal fungi are of particular interest. The effects of ecto-mycorrhizal (ECM) and arbuscular mycorrhizal (AM) fungi on the transport of uranium (U) or radiocaesium (Cs) were investigated both under pot and in vitro culture conditions. Results obtained in vitro demonstrated that AM hyphae can take up and translocate $U$ and Cs towards roots, while this uptake and translocation were not perceptible using pot culture systems with soil. These contrasting results could be due to different experimental conditions, including the $\mathrm{K}$ level in the external solution and the bio-availability of Cs. The in vitro studies also indicated that root colonisation by AM fungi might limit $\mathrm{U}$ and Cs root transport. Under pot culture conditions, they appeared to significantly reduce root to shoot translocation of $\mathrm{U}$. Under the same conditions, ECM transport of Cs was demonstrated, and appeared to be dependent on fungal species. A better estimation of the potential use of mycorrhizal fungi for the phytoremediation of $\mathrm{RN}$-contaminated areas is now available and will be further discussed.
\end{abstract}

\section{INTRODUCTION}

Over the last decades, contamination of the environment by radionuclides $(\mathrm{RN})$ has been observed in a large number of countries as a result of human activities such as mining, nuclear weapon production and testing, and nuclear accidents. Management of sites or larger areas, which have been exposed to either intense or diffuse RN pollution has become a major concern as they can constitute a serious risk for human health, provoke social stress and economic disturbances or even jeopardise ecosystem stability. Until recently, most remediation technologies concerned the removal of the topsoil or soil excavation and physico-chemical treatments. However, a growing interest is now rising towards the use of biological RN remediation strategies. The use of plants to remove RN from the environment or to render them harmless was proposed as a realistic alternative. Negri \& Hinchman [1] summarised data on the use of plants for treatment of ${ }^{3} \mathrm{H}, \mathrm{Pu},{ }^{90} \mathrm{Sr}, \mathrm{U}$ and ${ }^{137} \mathrm{Cs}$. If these results are promising, additional information on the role of soil micro-organisms in processes affecting $\mathrm{RN}$ fate (eg. solubilisation, complexation, sequestration, immobilisation, transformation, precipitation) should be investigated thoroughly to 
delineate efficient phytoremediation strategies. Of these micro-organisms, the mycorrhizal fungi, which are intimately associated to the root system of most plants, have been proposed as a key component of the soil-plant interface as they participate in the transport of various elements from soil to plants.

Within the framework of the "Nuclear Energy» programme of the European Commission, the project «MYRRH» (Use of MYcoRRHizal fungi for the phytostabilisation of radio-contaminated environment) was funded to decipher and understand the role and the influence of mycorrhizal fungi in the accumulation of RN (e.g. U and Cs) by plants. The present contribution intends to summarise the most prominent results obtained and outline the potential use of mycorrhizal fungi in phytoremediation strategies.

\section{ROLE OF MYCORHIZAL FUNGI IN URANIUM ACCUMULATION IN PLANT}

Since the late nineties, long-term rehabilitation of U-contaminated sites using phytoremediation techniques and associated microbiota has been considered as an interesting solution [2, 3]. However, very little information has been available on the effect of mycorrhizal fungi on $U$, the only consistent report being made by Weiersbye et al. [4]. These authors observed higher concentrations of $U$ in intraradical structures of an AM fungus as compared to host root tissues. Thus, a better understanding and quantification of the contribution of mycorrhizal fungi to U bio-availability appeared necessary as a pre-requirement to any phyto-remedial option involving these symbionts.

The first evidence that mycorrhizal fungi may participate in U plant bio-availability was given in a study performed under in vitro conditions using monoxenic cultures [5]. This model system was chosen to enable the quantification of $U$ uptake and translocation by an AM fungus, Glomus intraradices, without the influence of other micro-organisms or soil properties that would impair these observations. The monoxenic system consist of a two-compartment Petri plate divided by a plastic wall. On one side, i.e. in the culture compartment (CC), a root grown in symbiosis with an AM fungus. Following colonization, an extraradical mycelium develops that cross the partition wall and proliferate in the medium on the other side, i.e. in the hyphal compartment (HC). The HC was labelled with ${ }^{233} \mathrm{U}$ and various $\mathrm{pH}$ conditions were applied.

The results obtained demonstrated the uptake of $U$ by the AM fungus and its translocation towards its host root. Since the model system used was constituted of an excised host root, transfer to root cells could not be demonstrated. This additional information was obtained by performing an in vivo two-compartment experiment where transfer of $U$ to the root cells was shown (Jakobsen et al., unpublished data). Immobilisation of $U$ within the intraradical fungal structures was hypothesised by Rufyikiri et al. [5] and corroborated the earlier work of Weiersbye et al. [4].

In the same study [5], the importance of external $\mathrm{pH}$ on $\mathrm{U}$ speciation and uptake and translocation by AM fungi was shown (Table 1). Uranyl cations or uranyl sulfate species were translocated to a higher extent, while phosphate, hydroxyl and carbonate species were rather immobilised in AM extraradical structures. At pH 4, most exchange sites of the fungal extraradical structures were probably saturated by $\mathrm{H}^{+}$leading to a low $\mathrm{UO}_{2}{ }^{2+}$ adsorption by the hyphae, which might have participated in the low $\mathrm{U}$ concentration in hyphae and facilitated its translocation. Raising the $\mathrm{pH}$ to more neutral or alkaline values, which results in an increase of negative charge availability, did not affect $U$ sorption. At this $\mathrm{pH}$, $U$ species formed are either neutral or even negatively charged. Therefore, Rufyikiri et al. [5] suggested accumulation of $U$ following complexation or precipitation, specifically at $\mathrm{pH} 5.5$ where phosphate species were dominant.

In another experiment, the relative contribution of the AM fungal extraradical mycelium to the uptake and translocation of $U$ as compared to that by mycorrhizal or non-mycorrhizal roots were investigated [6]. The model used was similar to the one described above, but the $\mathrm{pH}$ of the media was adjusted to 5.5, and two additional treatments were added. A mycorrhizal root treatment where root and AM fungi were allowed to grow in both compartments, named root-hyphal compartment (RHC). A non-mycorrhizal root treatment where roots were allowed to grow in both compartments, named root compartment (RC). 
The results demonstrated a higher U concentration in extraradical fungal mycelium than in roots, when these had been in contact with the labelled medium (Table 1). The exchange sites could have contributed to the observed results, because the CEC of fungal extraradical mycelium was higher than the CEC of roots. However, the authors, by performing sequential extractions of $\mathrm{U}$ with $\mathrm{CuSO}_{4}$ and $\mathrm{HCl}$, noticed that only a small fraction of $U$ was released from the extraradical fungal structures or roots, thus demonstrating that the formation of stable complexes or precipitates was likely the main mechanism of $U$ accumulation. $U$ fixed on exchange sites was then suggested as starting point for complexation or precipitation reactions with anions. The outcome of such a mechanism could be a restricted $U$ translocation along fungal mycelium and $U$ root transport.

Table 1. Uranium concentrations $\left(\mathrm{Bq} \cdot \mathrm{g}^{-1} \mathrm{f} \mathrm{wt}\right.$ ) of roots and medium containing or not fungal biomass in the culture compartment (CC), fungal biomass in the hyphal compartment (HC) and root hyphal compartment (RHC) and root biomass in the RHC and root compartment (RC). Adapted from Rufyikiri et al. [6] with permission.

\begin{tabular}{|l|c|c|c|}
\hline & \multicolumn{3}{|c|}{ U concentration (Bq. - $^{-1} \mathrm{f}$ wt) } \\
\cline { 2 - 4 } & $\mathrm{HC}$ & $\mathrm{RHC}$ & $\mathrm{RC}$ \\
\hline Fungal biomass in labelled medium & $276 \mathrm{a}^{1}$ & $265 \mathrm{a}$ & - \\
\hline Roots in labelled medium & - & $49 \mathrm{~b}$ & $28 \mathrm{a}$ \\
\hline Medium and fungal biomass in CC & $0,41 \mathrm{~b}$ & $0,63 \mathrm{~b}$ & $0,04 \mathrm{~b}$ \\
\hline Roots in CC & $9,8 \mathrm{~b}$ & $10,9 \mathrm{~b}$ & $1,4 \mathrm{a}$ \\
\hline
\end{tabular}

${ }^{1}$ Within rows, averages followed by the same letter are not significantly different $(P=0.05)$.

The lower $\mathrm{U}$ concentration in the non-mycorrhizal roots in the RC versus the mycorrhizal roots in the RHC observed in this study is poorly understood. It might be due to a more active U uptake mechanisms due to the actual mycorrhizal status of the roots and/or to a marked contribution of the intraradical fungal structures to the accumulation of $U[4,5]$.

Fungal hyphae appeared more efficient to translocate $U$ than roots even if their total cross section area at the level of the partition between the two compartments was 281-fold lower than the cross section of roots. It has also to be noticed that $U$ concentrations in mycorrhizal roots of the CC were equivalent whether hyphae alone or with the contribution of roots participated in their accumulation of $U$. This suggest, as the number of hyphae crossing the partition wall was similar between the HC and RHC treatments, that hyphae probably contribute to the largest extend to the accumulation of $U$ in the roots of the CC in the RHC treatment.

Finally, a study conducted in soil with Trifolium subterraneum colonised by G. intraradices suggests that plant mycorrhization may decrease $U$ concentration in shoots of plants grown at high levels of $U$ [7] by reducing $U$ translocation from root to shoot. The results of this study also show that, at none of the $U$ concentrations applied, a difference was measured between $U$ root concentrations of mycorrhizal or non-mycorrhizal plants. Thus, if we consider that AM fungi participate in U uptake and translocation and that the intraradical fungal structures accumulate $U$ in the form of complexes or precipitates [5, 6], then it is possible to assume that direct U root uptake was impaired. Furthermore, by taking into account the results of Rufyikiri et al. [6], that $U$ may also form complexes or precipitates within the roots, $U$ root transport would be reduced and thus root to shoot translocation. It is also possible to envision that AM fungi might limit $U$ root transport and thus translocation towards the shoots. Explanation of these mechanisms might be found in the physiological interactions between the AM fungus and the host root cells. Indeed, following mycorrhizal colonisation, the regulation of gene expression encoding for root plasma membrane transporters of various elements is modified $[8,9]$. The down-regulation of the gene expression of transporters affecting $U$ uptake could have reduced the influx of $U$ in the root cells but also its efflux. 


\section{ROLE OF MYCORHIZAL FUNGI IN RADIOCAESIUM ACCUMULATION IN PLANT}

Several studies before and after the Chernobyl accident reported high amounts of Cs in fruit bodies of many ectomycorrhizal (ECM) fungi, while no literature has reported the transport of this element by ECM to their host plants. The role of AM fungi in Cs acquisition and accumulation by plants were also investigated, but most often yielded contradictory results (see [10] and references therein).

In an experiment (Leyval et al. unpublished data) conducted in a two-compartment pot system consisting of a culture compartment and a labelled hyphal compartment, it was demonstrated that two ECM fungi (Paxillus involutus and Suillus bovinus (Zn sensitive isolate)) were able to transport radiocaesium to their host plants, i.e. Pinus sylvestris, with concentration ranging from 48 to $311 \mathrm{~Bq} \cdot \mathrm{g}^{-1}$ in shoots and 147 to $406 \mathrm{~Bq} \cdot \mathrm{g}^{-1}$ in roots, while Suillus bovinus ( $\mathrm{Zn}$ tolerant isolate), Laccaria laccata and Telephora terrestris appeared unable to do so. In the same study, Cs root to shoot ratios showed that Cs remained mainly in the roots, suggesting a potential ability for phytostabilisation of this element in mycorrhizal roots. This should be confirmed by data comparing the relative contribution of roots and ECM hyphae to the accumulation of Cs in plants and Cs partition between root and shoot.

In 2003 [10], Declerck et al. unambiguously demonstrated that AM fungi could take up and translocate Cs in vitro using the two-compartment system of Rufyikiri et al. [5] (see above). The total uptake of Cs represented $1.5 \%$ of the initial Cs present in the hyphal compartment (HC). Of this Cs, $69 \%$ was translocated to the culture compartment (CC) and 55\% was measured in the roots. Following these results, Dupré de Boulois et al. [11] compared and quantified the respective contribution of the extraradical hyphal network, roots and, both in combination, on Cs uptake and translocation. In this study, the uptake of ${ }^{134} \mathrm{Cs}$ by extraradical hyphae represented $5.2 \%$ of the initial ${ }^{134} \mathrm{Cs}$ added in the HC and ${ }^{134} \mathrm{Cs}$ activity measured in these roots of the CC, accounted for $78.1 \%$ of the total ${ }^{134} \mathrm{Cs}$ taken up by the hyphae (Table 2). Correlation analyses showed that uptake and Cs root content were positively correlated with hyphal density and therefore indicated that AM fungi may have an influence on Cs immobilisation and/or in Cs plant accumulation.

The total uptake of Cs by mycorrhizal roots and hyphae in the RHC treatment and by the roots alone in the RC treatment was higher than the total uptake by hyphae alone in the HC treatment (33.3, 32.8 and $5.2 \%$ of the initial ${ }^{134} \mathrm{Cs}$ activity, respectively). Nevertheless, the Cs taken up in the RHC and RC treatments mostly remained in the roots of these compartments (19.2 and 16.5\%, respectively) whereas extraradical fungal hyphae translocated $80.8 \%$ of the Cs taken up towards the roots of the CC.

Table 2. Radiocaesium contents (Bq.Petri plate ${ }^{-1}$ ) of roots and medium containing or not fungal biomass in the culture compartment (CC), fungal biomass in the hyphal compartment (HC) and root hyphal compartment (RHC) and root biomass in the RHC and root compartment (RC). Numbers in parentheses are percentages that represent the radiocaesium distribution within a treatment. Adapted from Dupré de Boulois et al. [11] with permission.

\begin{tabular}{|l|c|c|c|}
\hline & \multicolumn{3}{|c|}{ Cs content (Bq.Petri plate ${ }^{-1}$ ) } \\
\cline { 2 - 4 } & $\mathrm{HC}$ & $\mathrm{RHC}$ & $\mathrm{RC}$ \\
\hline Solution (EC's) & $10135(95) \mathrm{ab}$ & $7158(67) \mathrm{b}$ & $7203(67) \mathrm{b}$ \\
\hline Hyphae (EC's) & $37(0.35) \mathrm{a}$ & $19(0.18) \mathrm{a}$ & - \\
\hline Root in the EC's & - & $2783(26) \mathrm{a}$ & $2878(27) \mathrm{a}$ \\
\hline Gel with fungal biomass (CC) & $64(0.60) \mathrm{a}$ & $78(0.72) \mathrm{a}$ & $43(0.40) \mathrm{a}$ \\
\hline Roots in the CC & $454(4.2) \mathrm{a}$ & $692(6.4) \mathrm{a}$ & $578(5.4) \mathrm{a}$ \\
\hline
\end{tabular}

The rather low Cs root translocation from the RHC or RC to the CC's is poorly understood. A possible explanation could be the absence of a strong sink driving force such as an aerial part or the presence of actively growing root meristems in the RHC and RC. A possible accumulation of Cs in the root cells to maintain cell osmotic potential [12] could also be possible. Indeed, the lack of $\mathrm{K}$ in the medium of the labelled compartments could have resulted in its substitution by Cs and thus restricted the transport of Cs towards the xylem. The authors suggested that this could be linked to the inhibition of the KOR (potassium outward-rectifying) channels. The KOR channels mediate the 
majority of the efflux of $\mathrm{K}^{+}$and $\mathrm{Cs}^{+}$from the roots cells, but as no $\mathrm{K}^{+}$was provided in the labelled compartments, the probability of openness of the KOR channels was probably close to zero to prevent $\mathrm{K}$ and thus Cs leakage from the root cells [13]. Moreover, Pilot et al. [14] observed that in K-starved plants, the expression of genes encoding for a KOR channel in Arabidopsis thaliana was reduced. Consequently, following the influx of Cs in root cells through the $\mathrm{K}^{+} / \mathrm{H}^{+}$symporters or through the VIC (voltage-insensitive cation) channels [15], the limitation of the efflux of Cs would lead to its accumulation in the root cells [16]. The reduction of Cs loading in the xylem would therefore result in the decrease of the amount of Cs translocated along the roots. For instance, Buysse et al. [17] and Smolders et al. [18] observed that the root/shoot Cs concentration ratio increased with decreasing K supply, thus supporting this hypothesis.

Dupre de Boulois et al. (submitted) also observed that translocation of Cs within mycorrhizal roots could be impaired by the mycorrhization of the roots and could be directly linked to the colonization of roots . A possible explanation is the down-regulation of K/Cs plasma-membrane transporters of roots cells. Indeed, as AM colonization appears and spreads, interactions between the partners of the symbiosis, lead to various physiological modifications among both. One of this interaction is the effect of the AM fungi on their host plasma membrane transporter gene expression [8, 9]. Consequently, it is possible to envision that a down-regulation of genes encoding for K/Cs transporters at the level of the root cell plasma membranes could be triggered by the establishment of AM symbiosis and impair Cs root transport. The consequence of such processes could therefore result in the reduction of root to shoot translocation of Cs in mycorrhizal plants.

Three separate experiments performed by three laboratories using different protocols and fungus/host plant combinations [19] to investigate whether AM fungi can transport Cs in soil-based systems. Here only the first experiment will be discussed. The system consisted of two chambers, similar to the one used for Cs transport by ECM (see above). The culture compartments were filled with a substrate consisting of sand/expanded clay/peat/clay loam soil 2:1:1:1 by volume. Plants were watered daily with a $\mathrm{K}(1 \mathrm{mM})$ and $\mathrm{P}(0.1 \mathrm{mM})$ nutrient solution throughout the experiment (70 days). The hyphal compartment was labelled with ${ }^{137} \mathrm{Cs}$, but also with ${ }^{32} \mathrm{P}$ as a second radio-tracer which AM fungi are known to transport. The results of this experiment, and the two similar ones run by two other laboratories, showed that no Cs was transported or even translocated by two AM fungi, while ${ }^{32} \mathrm{P}$ was transported, proving that extraradical hyphae were present and active in the HC. The authors concluded that because the uppermost soil in agricultural ecosystems often contains ample amounts of clay, and that application of K-fertilizers is common, AM fungi were highly unlikely to play any significant role in plant Cs accumulation. However, this study did not rule out that AM can transport Cs to plants in other ecosystems where clay levels (e.g. frayed edge sites) and $\mathrm{K}$ concentrations are lower. On the other hand, as in vitro experiments were performed without $\mathrm{K}$ in the labelled medium and a low initial $\mathrm{K}$ concentration $(0.18 \mathrm{mM})$ in the culture compartment, and the soil-based experiments were conducted with an excess of $\mathrm{K}$, additional studies are needed where intermediate and realistic K concentrations and low amounts of frayed edge sites (FES), in soil based experiments, are employed. However, results of both in vitro and soil-based studies are promising for the use of mycorrhizal fungi in phytoremediation strategies. Indeed, these plant-health promoting fungi do not contribute to plant Cs accumulation in high K concentrations and in presence of high amounts of FES and might limit Cs root to shoots translocation in case of Cs root uptake or AM fungi-plant transfer.

\section{CONCLUSION}

Over the last decade, phytoremediation has received a growing interest as a potential alternative for the management of cultivated and semi-natural ecosystems as well as former industrial sites, which have been exposed to RN pollution. The goal of the MYRRH project was to understand and evaluate the role and the influence of mycorrhizal fungi in the $\mathrm{RN}$ accumulation by plants in order to determine whether these micro-organisms should be considered in future phytoremediation strategies. From the results obtained on U and Cs, a potential use of mycorrhizal fungi could be envisioned. For U, both in vitro and 
in vivo studies yielded the same conclusion, being that AM fungi can significantly reduce root to shoot translocation. In the case of Cs, additional studies may be required. Indeed, if the physiological ability of AM fungi in the uptake and translocation of Cs, and the possible influence of AM symbiosis in Cs root transport has been determined, it appears that soil characteristics (e.g. presence of frayed edges sites, especially) and concentration of $\mathrm{K}$ in the soil solution might influence their role. Nevertheless, it should be mentioned that when these parameters are too high, even plants would not accumulate Cs. Therefore, the role of mycorrhizal fungi in Cs accumulation by plants should be investigated under a wider range of soil conditions. Finally, further studies will have to be performed to delineate the potential role of ECM fungi, but preliminary results on Cs appear encouraging.

\section{Acknowledgements}

This work was supported by the EU contract FIGE-CT-2000-00014 entitled "Use of mycorrhizal fungi for the phytostabilisation of radio-contaminated environments". H.D.D.B. acknowledges receipt of a FRIA fellowship via the FNRS and S.D. the financial support from the Belgian Federal Office for Scientific, Technical and Cultural affairs (contract BCCM C3/10/003).

\section{References}

[1] Negri M.C. and Hinchmann R.R., Using Plants to Clean Up the Environment (Wiley-Interscience, John Wiley and Sons, Inc. New york, NY, 2000) pp. 107-132.

[2] Huang J.W., Blaylock M.J., Kapulnik Y. and Ensley B.D., Environ. Sci. Technol 32 (1998) 2004-2008.

[3] Shahandeh H., Lee J.H., Hossner L.R. and Loeppert R.H., Fate of trace elements in the rhizosphere (CRC Press LLC, London, 2001) pp. 93-124.

[4] Weiersbye I.M., Straker C.J. and Przybylowicz W.J., Nucl. Phys. B 158 (1999) 335-343.

[5] Rufyikiri G., Thiry Y., Wang L., Delvaux D. and Declerck S., New Phytol. 156 (2002) 275-281.

[6] Rufyikiri G., Thiry Y. and Declerck S., New Phytol. 158 (2003) 391-399.

[7] Rufyikiri G., Huysmans L., Wannijn J., Van Hees M., Leyval C. and Jakobsen I., Environ. Pollution 130 (2004) 427-436.

[8] Burleigh S.H. and Bechmann I.E., Plant Soil 244 (2002) 247-251.

[9] Ferrol N., Barea J.M. and Azcon-Aguilar C., Plant Soil 244 (2002) 231-237

[10] Declerck S., Dupré de Boulois H., Bivort C. and Delvaux B., Environ. Microbiol. 5 (2003) 510-516.

[11] Dupré de Boulois H., Delvaux B. and Declerck S., Environ. Pollution In press.

[12] Leigh R.A. and Wyn Jones R.G., New Phytol. 97 (1984) 1-13.

[13] Maathuis F.J.M. and Sanders D., J. Exp. Bot. 48 (1997) 451-458.

[14] Pilot G., Gaymard F., Mouline K., Chérel I. and Sentenac H., Plant Mol. Biol. 51 (2003) 773-787.

[15] White P.J. and Broadley M.R., New Phytol. 147 (2000) 241-256.

[16] Zhu Y.G., Shaw G., Nisbet A.F. and Wilkins B.T., Ann. Bot. - London 84 (1999) 639-644.

[17] Buysse J., VandenBrande K. and Merckx R., Plant Soil 178 (1996) 265-271.

[18] Smolders E., Kiebooms L., Buysse J. and Merckx R., Plant Soil 181 (1996) 205-209.

[19] Joner E.J., Roos P., Jansa J., Frossard E., Leyval C. and Jakobsen I., Appl. Environ. Microb. In press. 